\section{SUBJECT INDEX - VOLUME 36, $1994^{1}$}

Accelerator mass spectrometry (AMS), 237-246, 247250, 399-405, 407-412

Adriatic Sea, $136-137$

Africa, 281-302

Alps, 345-357

Antarctica, 173-185

Archaeological dating, 51-57, 59-71, 95-111, 221-236, 247-250, 257-279, 281-302, 345-357, 359-366, 367-376, 377-389, 391-398

Arctic Ocean, 327-343

Argon ( $\left.{ }^{39} \mathrm{Ar}\right), 327-343$

Australia, 73-94

Authors, Instructions to, $159-168$

Becker, Bernd (obituary), v-viii(1)

Benzene synthesis, 47-49

Bosnia and Herzegovina, 316-317, 320

Britain, 27-35, 95-106

Bronze Age, 266-270, 359-366

CALHIS program, $1-10$

Calibration, 1-10, 11-26, 221-236

Calibration curves, 1-10, 11-26, 27-35, 221-236

Calibration stochastic distortion, 221-236

Chalcolithic, 391-398

Charcoal, 51-57

China, 37-45, 47-49

Climate change, 37-45, 59-71, 173-185, 187-202, 203219

Coastal morphogenesis, 59-71

Cook Islands, 59-71

Counting vials, 47-49

Croatia, 203-219, 303-307, 308-310, 317-319, 322323

Cyprus, 106

Czech Republic, 322

$\delta^{13} \mathrm{C}, 27-35$

Deep water, 327-343

Deglaciation, 345-357

Dendrochronology, 27-35

$\Delta R, 51-57$

Egypt, 281-294, 297-298, 300

Estonia, 157-158

Fontes, Jean-Charles (obituary), $\mathrm{ix}-\mathrm{x}(1)$

France, 345-357

Gaussian distributions, 1-10

Ghana, 298-299

Glacial period, $187-202$

Glaciation, 173-185, 345-357

\section{Hawaii, 51-57}

Histograms, ${ }^{14} \mathrm{C}, 1-10,11-26,27-35$

Holocene, 37-45, 51-57, 59-71, 173-185, 187-202, 203-219; Holocene regression, 59-71

Ice Man, 247-250

India, 106-109, 251-256

Inorganic carbon, 237-246

Intercalibration, 251-252

International Collaborative Study, 131-132

Irish oak, 27-35

Iron Age, 270-272, 297-300

Isolation ages, 327-343

Israel, 391-398

Italy, 109, 133-142, 143-147, 247-250

Jordan, 109-110

KORHIS program, 1-10

Lake water, 173-185

Lake sediments, 173-185, 187-202, 203-219

Lake-level changes, 187-202

Latvia, 153-157, 377-389

Libya, 151-152, 295-296

Limestone coasts, 51-57

Limnology, 73-94

Liquid scintillation spectroscopy, 47-49

Lithuania, 158

Loess, 37-45

Magnetic susceptibility, 37-45

Mali, 299-300

Maori, 221-236

Marine calibration curve, 221-236

Marine carbon, 173-185

Marine sediments, $136-137$

Marine shells, 51-57, 221-236

Mesolithic, 257-260

Middle Ages, 272-276

Montenegro, 321

$\mathrm{Na}_{2} \mathrm{CO}_{3}, 237-246$

Nahal Issaron, 391-398

Negev Desert, 391-398

Neolithic, 260-266, 282-297, 359-366, 391-398

New Zealand, 221-236

Novgorod, 377-389

Oak, 27-35

Ötztal Ice Man, 247-250

Pacific Ocean, 51-57

Paleolithic, 257-260, 281-282, 345-357, 367-376

${ }^{1}$ Page numbers are to inclusive articles except in the case of geographical regions in date lists. 
Paleosol, 37-45

Peat, $407-412$

Penguin guano, 173-185

Philippine Islands, 131

Pleistocene, 51-57

Poland, 187-202, 257-279

Pollen, 407-412

Polynesia, 59-71

Pre-Pottery Neolithic B (PPNB), 391-398

Pretreatment, see Sample preparation

Reservoir effect, 51-57, 173-185, 187-202

Russia, 359-366, 367-376, 377-389

Sample preparation, 47-49, 237-246, 247-250, 251, $399-405,407-412$

$\Sigma \mathrm{CO}_{2}, 237-246$

Sea-level change, $59-71$

Seawater, 237-246

Sediments, 173-185

Serbia, 308, 310-316, 320-321

SI units, use in RADIOCARBON, 160

Siberia, 367-376

Siegenthaler, Ulrich (obituary), v-viii(3)
Slovenia, 307-308, 310, 319-320

Smoothing of ${ }^{14} \mathrm{C}$ curves, $1-10,11-26$

Soils, 37-45

Southern hemisphere, 221-236

Stable isotopes, 27-35, 237-246

Staraya Lagoda, 377-389

Statistical analysis, 23-26

Sudan, 150-151, 296-297

Suess, Hans E. (obituary), xi-xv(1)

Taiwan, 113-131

Tephra chronology, 173-185, 407-412

Travertine, 203-219

Turkey, 147-149

Uranium-series dating, 203-219

Volcanic coasts, $51-57$

"Wiggles", 221-236

World Ocean Circulation Experiment, 237-246

Würmian glaciation, 345-357

Younger Dryas, 187-202 